\title{
Management of congenital missing maxillary incisors by orthodontic treatment followed by fixed prosthesis
}

\author{
Hossain MZ1', BDS, PhD; Haque S², BDS,MS; Mamun MSA³, BDS
}

\begin{abstract}
:
Fixed prosthetic restoration has become a primary treatment option for the replacement of congenitally missing lateral incisors where adequate Implant restorations have limited use due to many factors. The central incisor and canine often erupt less than optimal positions adjacent to the edentulous lateral incisor space, and therefore preprosthetic orthodontic treatment is frequently required. Derotation of the central incisor and canine, space opening and correction of root proximities may be required to create appropriate space, in which fixed prosthesis may achieve an esthetic restoration. This paper presents two case reports with congenital missing lateral incisors and one case report with missing central incisors due to trauma. The paper also discusses the aspects of preprosthetic orthodontic diagnosis and the treatment that needs to be considered with conservative and fixed prosthetic replacement .
\end{abstract}

Key Words: Congenital missing lateral incisors, traumatic missing central incisors, preprosthetic orthodontics, fixed prosthesis. (Ban J Orthod and Dentofac Orthop, Oct 2011; Vol-2, No. 1, p 16-19)

\section{INTRODUCTION:}

Congenital missing maxillary lateral incisors and central incisors present challenging treatment planning and mechanotherapy problems for orthodontists, prosthodontists and general practitioners. The treatment approach that is commonly taken is creating adequate space to replace the missing lateral incisors. In this article, in-addition to describing the management of malocclusions resulting from congenitally missing maxillary lateral incisors (Case 1 and Case 2), we will also describe the management of missing central incisors due to trauma (Case 3). In the later case, a considerable amount of space were lost, where we had to regain space orthodontically to make a fixed prosthesis to simulate central incisors.

The successful use of dental prosthesis to replace missing teeth has been one of the most evolving areas of clinical dentistry for many decades. ${ }^{1}$ At a time when aesthetic dentistry has gained prominence, permanent prosthodontic solutions such as implants have become optimal esthetic treatment options. ${ }^{1,2}$ While implants have expanded restorative treatment options, treatment planning has become more complex for the dental practitioner, and an interdisciplinary team approach is recommended. $2,3,4$ However, implant technology is yet to be estabished in our country for many reasons. Thus, an interdisciplinary approach may involve preprosthetic orthodontic treatment. Orthodontic alignment will facilitate proper fixed prosthesis. ${ }^{2}$

\section{CASE SUMMARY and ORTHODONTIC TREATMENT PLANNING and RESULTS:}

Case 1 and case 2:

Both the cases had median diastema. Overbite and overjet were about $1 \mathrm{~mm}$, having tendency of edge to edge bite due to collapse of the arch for congenital missing laterals. The aim of treatment was to close the median diastema, adequate space opening for

fixed prosthesis of laterals. This will cause proclination of upper centrals which will help in giving normal over bite and over jet.

Treatment alternatives for restoring spaces resulting from congenitally missing laterals include removable partial dentures, conventional fixed bridges, resin-bonded bridges, autotransplantation, orthodontic repositioning of canines to close the edentulous space, and single-tooth implant. ${ }^{5,6}$ Although adjacent teeth may have to be repositioned orthodontically to create adequate space for any prosthesis.

When there is a history of congenitally missing teeth, (Case 1, Case 2) asymmetric loss of primary teeth, over-retention of deciduous lateral incisors and canines, lack of developmental canine bulge, or impacted maxillary canines, the possibility of missing lateral incisors should be immediately investigated. 7,8

CASE 1. ( Fig. 1 A,B. Fig. 2 A,B,C: 3 A,B)

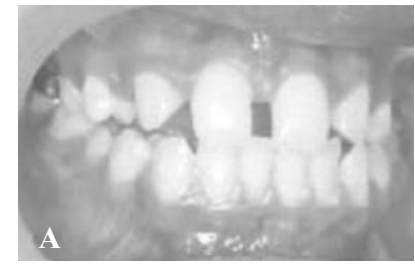

Fig.1A : Congenital Missing upper laterals with midline diastema

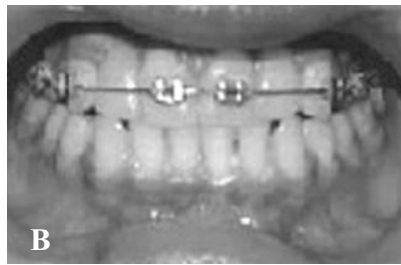

Fig.1B : Orthodontic approximation of upper centrals and space opening for laterals. Mock trial with removable partial denture

${ }_{1}^{1}$ Professor and Head, Dept of Orthodontics and Dentofacial Orthopedics, Dhaka Dental College and Hospital, Mirpur -14, Dhaka 1206. ${ }^{2}$ Assistant Professor, Dept. of Conservative Dentistry, Dhaka Dental College and Hospital ${ }^{3}$ FCPS, Part II Trainnee, Dept. of Orthodontics and Dentofacial Orthopedics, Dhaka Dental College. 

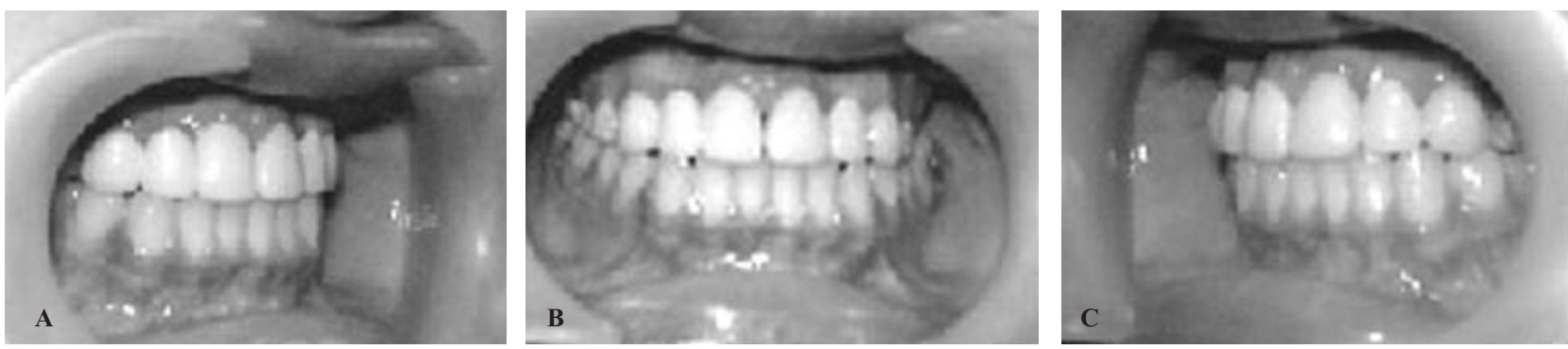

Fig: 2 (A,B,C) : A. Removable partial denture has been replaced by fixed prosthesis B. Right Lateral view C. Left Lateral View
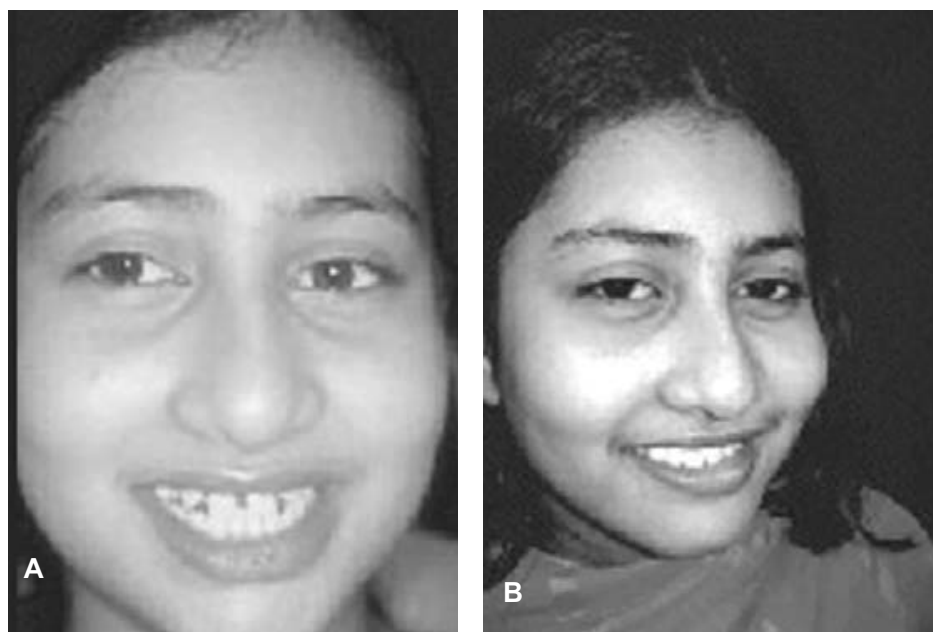

Fig. 3 ( A,B), Facial Photo : A. Smile: Before treatment, spacing due to missing laterals are visiable B. Smile: After orth-prostho treatment, has greatly improved.

CASE:2: Fig. 1 A,B, C; Fig. 2 A, B
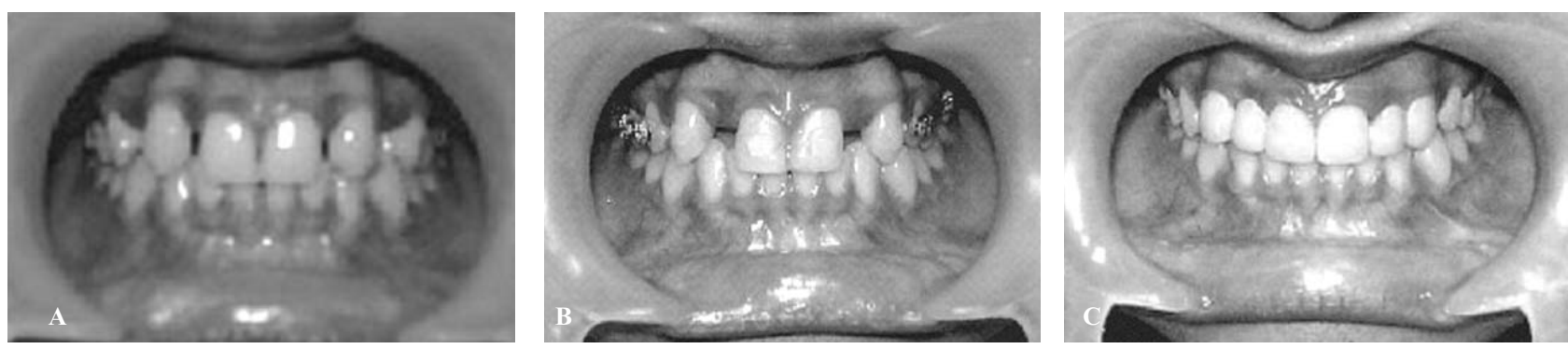

Fig. 1 A, B, C : A.congenital missing laterals B. space opening for prosthesis C. After Fixed Prosthesis
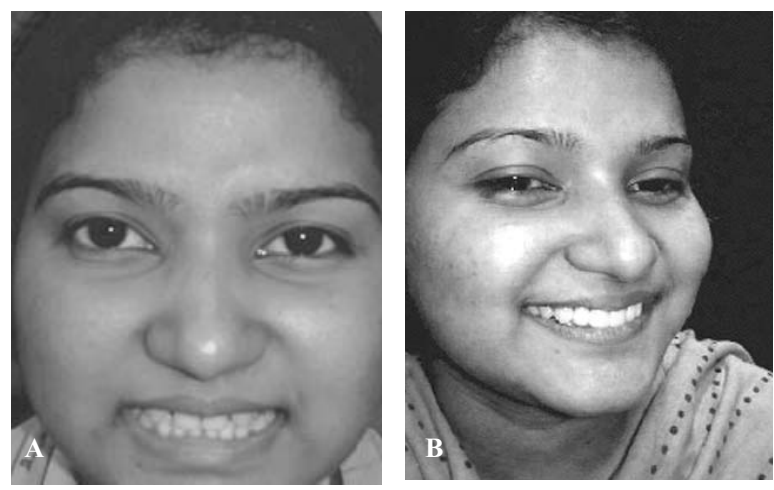

Fig. 2 (A, B): A. Smile before treatment

B. Smile after orthodontic treatment followed by fixed prosthesis 
The above two cases with congenital missing, early investigation was especially important due to the higher association of congenitally missing or peg-shaped lateral incisors. ${ }^{9}$ In addition, early investigation will give the patient time to explore all possible treatment options. A full set of orthodontic records including radiographs, models and clinical photographs are recommended for the diagnosis of congenitally missing laterals and to planning of the preprosthetic orthodontic alignment. A diagnostic set-up model is also beneficial for planning of treatment and esthetics for patient motivation. ${ }^{7,9}$ Participating clinicians, orthodontist and prosthodontist should determine the patient's treatment plan collaboratively and communicate throughout the course of treatment to ensure all aspects of treatment are considered and the overall treatment objectives are achieved. ${ }^{10}$

CASE 3: A case with missing upper central incisors

Overview of the occlusal features: Missing centrals due to trauma, irregular arch form, crowding in both arches. Patient was using conventional partial denture which was very unpleasant for this young girl. (Fig. 1, 2) .

Treatment Plan: Extraction of lower 2nd premolars, leveling and alignment of both arches using edge-wise appliance.
Once the permanent central incisor and canine have been positioned orthodontically to create adequate mesio-distal space between the crowns and the roots of the teeth, orthodontic retention is necessary to maintain this space and the position of the teeth. While the braces are in place, an acrylic denture tooth with a bonded bracket can be ligated to the arch wire to further maintain space and improve esthetics. Following removal of the fixed appliances, conventional types of orthodontic retainers (Hawley retainers in the form of partial denture) can be used to maintain the space until the fixed prosthesis is placed and restored (case1Fig. 1B)

Coordination of both arches and reassessment of remaining upper arch space to accommodate centrals by prosthesis (3A). Thus, remaining space between the lateral incisor in the upper arch was redistributed orthodontically by slight approximation of laterals mesially (Fig: 3B) to prepare porecelain crown over laterals to simulate centrals ( Fig. 4, Fig.5). This resulted improvement of aesthetics by providing better smile line (Fig: 6).

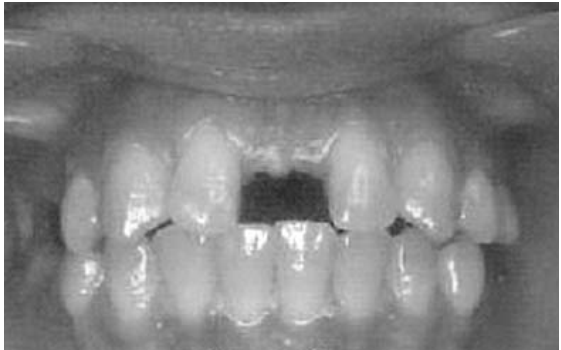

Fig: 1, Missing centrals due to trauma, with space loss

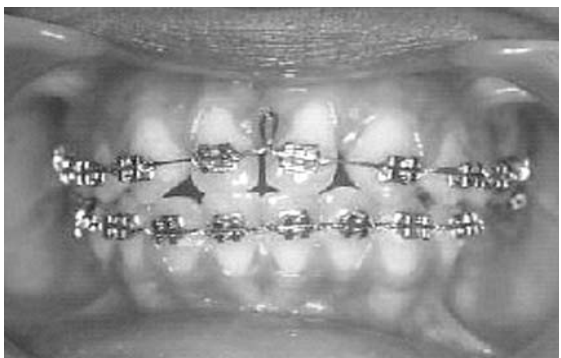

Fig 4, Space distribution for full Veneer Porcelain crown preparation

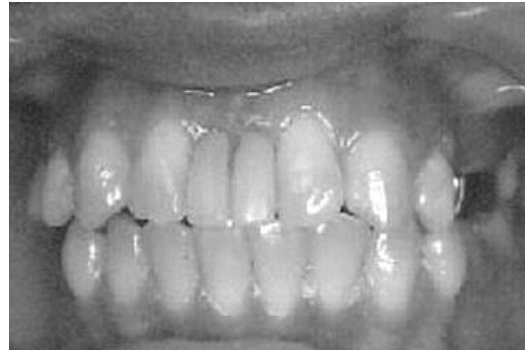

Fig: 2, Patient used removable Partial denture, not accepted aesthetically

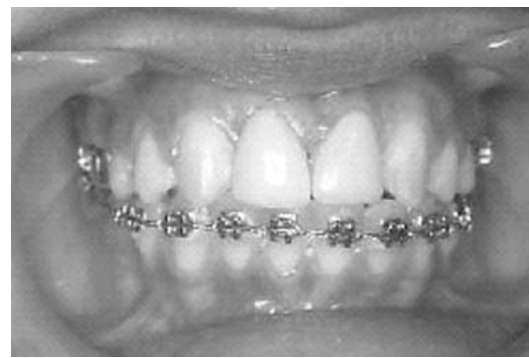

Fig..5. Full veneer porcelain crown over laterals Note the improvement as compared with Fig. 4

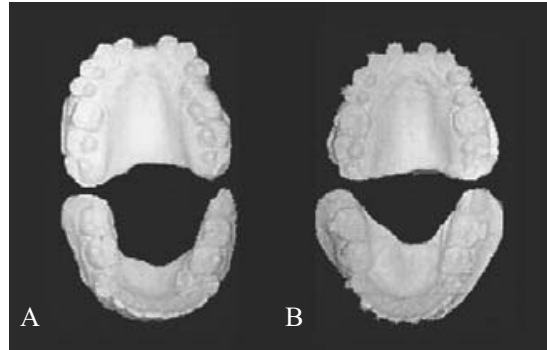

Fig;3 Plaster Model ( Before - A) and ( After-B) Orthodontic Alignment and space closure to reassess for full veneer crown over Laterals to simulate centrals

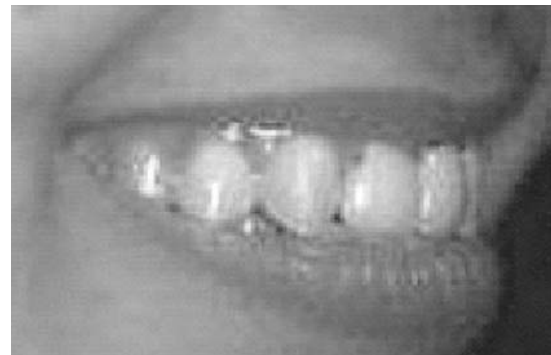

Fig. 6: Restored aesthetics and smile after Orthodontic alignment Followed by fixed prosthesis 


\section{DISCUSSION:}

For patients with congenitally missing lateral incisors, in addition to over-retained primary teeth, permanent canines may erupt or drift mesially into the edentulous space. If the space is to be opened orthodontically for ideal prosthesis, the canines will need to be moved distally, which may result in development of the alveolar ridge in the canine region. ${ }^{11}$ In cases where the occlusion and esthetics of the canine in the lateral position are acceptable, closure of the lateral space by the mesially positioned canine may be the simplest alternative treatment option. However, in all the above congenital missing cases (Case: 1, 2) we considered space opening followed by fixed prosthesis would be more acceptable on aesthetic point of view. The case with missing centrals (Case-3) were treated by coordinating both the arches followed by porcelain cap over laterals to simulate central which also restored patient's aesthesis, smile and function (Fig.4,5,6)

Esthetics as well as occlusion must be considered in the final orthodontic positioning of the teeth adjacent to the edentulous space. To satisfy the "golden proportion" principle of esthetics, the space for the maxillary lateral incisor should be approximately two-thirds of the width of the central incisor.11,12,13 However, if the patient is missing only one maxillary lateral incisor, the space required to achieve symmetrical esthetics and occlusion is primarily dictated by the width of the contralateral incisor.

The optimal time for placement of fixed prosthesis is after the growth of the maxilla, mandible and alveolus is complete. 11 If fixed prosthesis/implant are placed before growth is complete, the surrounding alveolar bone may continue to develop vertically and adjacent teeth may continue to erupt. Thus a discrepancy between the gingival margins of the prosthesis and the natural teeth is created and the prosthesis appears to became submerged. 11 This creates a functional as well as an esthetic problem.

\section{CONCLUSION:}

Congenital missing maxillary lateral incisors or missing central incisors or any coexisting malocclusion must be managed within an overall treatment plan. Factors relating to the patient's age; the size, shape, position and color of the teeth; the effect on occlusion; and overall facial and dental esthetics should be considered when deciding on whether to create an orthodontic space opening or space closure. Advantages and disadvantages of both treatment modalities and the various options for prosthetic replacements in an orthodontic space opening should be discussed with the patient. Creating an orthodontic space-opening followed by prosthetic solution was the conventional bridge. The advent of conservative prosthetic alternatives and the modern trend in orthodontics have made orthodontic space redistribution in case of missing centrals with replacing the incisors by making porcelain cap over laterals to simulate central incisors, which resulted improvement of aesthetics and function of the individuals. In all the cases smile of the patient was significantly improved (Fig. 3B, Case -1; Fig. 3B, Case- 2; Fig. 6 Case-3). Thus, fixed conservative-prosthesis is treatment of choice for most patients with congenitally missing laterals or missing centrals due to trauma where an implant technique is not available. Fixed prosthesis will maintain tooth continuity and provide esthetics and function. However, successful restorative treatment depends on interdisciplinary treatment planning, especially if preprosthetic orthodontic tooth alignment is required similar to these case reports.

\section{REFERENCES:}

1. Carlson H. Suggested treatment for missin lateral incisor cases. Angle Orthod 1952;22:205-16.

2. Tuverson DL. Orthodontic treatment using canines in place of missing maxillar lateral incisors. Am J Orthod 1970;58(2):109- 27.

3. McNeill RW, Joondeph DR. Congenitally absent maxillary lateral incisors: treatment planning considerations. Angle Orthod 1973;43:24-9.

4. Roth PM, Gerling JA, Alexander RG. Congenitally missing lateral incisor treatment. J Clin Orthod 1985;19:258-62.

5. Woodworth DA, Sinclair PM, Alexander RG. Bilateral congenital absence of maxillary lateral incisors: a craniofacial and dental cast analysis. Am J Orthod 1985;87:280-93./

6. Kharbanda OP. Orthodontics : Diagnosis and management of malocclusion and Dentofacial deformities

7. Asher $\mathrm{C}$, Lewis $\mathrm{DH}$. The integration of orthodontic and restorative procedures in cases with missing lateral incisors. $\mathrm{Br}$ Dent $\mathrm{J}$ 1986;160(7):241-5.

8. Biggerstaff RH. The orthodontic management of congenitally absent maxillary lateral incisors and second premolars: a case report. Am J Orthod Dentofacial Orthop 1992;102(6):537-45.

9. Millar BJ, Taylor NG. Lateral thinking: the management of missing upper lateral incisors. Br Dent J 1995;179(3):99-106.

10. Lodter C. Canines et agénésies des latérales supérieures. [Canines and agenesis of upper laterals.] Rev Orthop Dento Faciale 1995;29(4):487-94.

11. Schwaninger B, Shaye R. Management of cases with upper incisors missing. Am J Orthod 1977;71:396-405.

12. Levin EI. Dental esthetics and the golden proportion. J Prosthet Dent 1978; 40(3):244-52.

13. Fields HW Jr. Orthodontic-restorative treatment of relative mandibula anterior excess tooth size problems. Am J Orthod 1981;79(2):17683 .

\section{Correspondence:}

\section{Prof. Dr. Md. Zakir Hossain, BDS, PhD}

Prof. and Head,

Dept. of Orthodontics and Dentofacial Orthopaedics,

Dhaka Dental College and Hospital, Mirpur-14, Dhaka 1206.

E-mail:hossainzortho@yahoo.com,

web:www.orthodentalcare.com 